\title{
Improved model for the establishment and evaluation of detrusor overactivity in female Wistar rats
}

\author{
Nian-zhao Zhang ${ }^{1}$, Lin Ma', Jian-bo Zhang², Jun Chen ${ }^{1}$ \\ ${ }^{1}$ Department of Urology, Qilu Hospital, Shandong University and ${ }^{2}$ Department of Pathology, Shandong \\ Tumor Hospital, Jinan, P.R. China
}

\section{ABSTRACT}

Objective: To improve the model for establishment and evaluation of detrusor overactivity in female Wistar rats.

Materials and Methods: We ligated the perineal urethra of female Wistar rats and then performed filling cystometry. The probability of detrusor overactivity, bladder capacity, peak voiding pressure and histological changes were investigated.

Results: Detrusor overactivity ratio of the obstruction group was 32.4\%. Bladder capacity increased from $0.273 \pm 0.036 \mathrm{~mL}$ in control group to $0.89 \pm 0.19 \mathrm{~mL}$ in detrusor overactivity group $(\mathrm{P}<0.001)$, and peak voiding pressure increased from $45.9 \pm 4.1$ $\mathrm{cm} . \mathrm{H}_{2} \mathrm{O}$ to $63.5 \pm 17.4 \mathrm{~cm} . \mathrm{H}_{2} \mathrm{O}(\mathrm{P}=0.007)$. For obstruction group, compared to no detrusor overactivity rats, detrusor overactivity rats had higher bladder capacity $(0.89$ $\pm 0.19 \mathrm{~mL}$ versus $0.43 \pm 0.09 \mathrm{~mL}, \mathrm{P}<0.001)$ and higher peak voiding pressure $(63.5 \pm$ $17.4 \mathrm{~cm} . \mathrm{H}_{2} \mathrm{O}$ versus $\left.44.8 \pm 6.2 \mathrm{~cm} . \mathrm{H}_{2} \mathrm{O}, \mathrm{P}=0.005\right)$. Detrusor overactivity rats were classified according to peak voiding pressure $\left(49.2 \pm 4.2 \mathrm{~cm} . \mathrm{H}_{2} \mathrm{O}\right.$ versus $80.8 \pm 7.1 \mathrm{~cm} . \mathrm{H}_{2} \mathrm{O}, \mathrm{P}$ $<0.001)$. Moreover, bladder weight increased significantly in detrusor overactivity rats $(\mathrm{P}=0.003, \mathrm{P}=0.028)$ and detrusor histological hypertrophy was observed.

Conclusions: Ligating perineal urethra and filling cystometry with intra-urethral cannula approach is a simple and easily reproducible method to establish and evaluate the model of detrusor overactivity in rats.

\section{ARTICLE INFO}

Key words:

Lower Urinary Tract Symptoms;

Urinary Bladder; Urodynamics

Int Braz J Urol. 2014; 40: 414-22

Submitted for publication:

November 12, 2012

Accepted after revision:

September 18, 2013

\section{INTRODUCTION}

Detrusor overactivity (DO) is an urodynamic observation characterized by involuntary detrusor contractions during the filling phase (non-voiding contractions). These contractions, which may be spontaneous or provoked, produce a wave form on the cystometrogram, of variable duration and amplitude. The contractions may be phasic or terminal $(1,2)$. D0 may occur in patients with bladder outlet obstruction (B00), which can be caused by urethral strictures, congenital malformations such as posterior urethral valves, and more commonly, benign prostatic hyperplasia. B00 leads to several structural and functional changes in the detrusor muscle, and the adaptive growth does not fully restitute bladder function as BOO is frequently accompanied by the non-voiding contractions which can lead to clinical symptoms such as urinary frequency, urgency, and nocturia $(3,4)$. Recently, it has been reported that B00 is partially responsible for D0 because the prevalence of $\mathrm{DO}$ is associated with the degree of obstruction, and the treatment of BOO is potentially able to reverse the bladder wall changes and DO $(5,6)$. 
The most common method to create a model of DO rats involved reduction of the urethral diameter by placing a suture around the urethra (7), and significant increases were demonstrated in voiding frequency, voiding pressure and bladder capacity. Moreover, DO rats showed a pronounced "non-voiding contractions" during cystometry, and the categorization of different types of DO in conscious rats has been described (3). However, many disadvantages presented in the model of DO rats established in the previous studies. For example, D0 rats were established by partial obstruction with a method of transabdominal pathway, and the abdomen was opened through a midline incision (7). The architecture of cavitas pelvis was dissected, and the trauma might induce impairment of nerves and deformity of bladder which might influence the function of bladder. Therefore, in the study, we improved the method of establishment and evaluation of DO model in female Wistar rats.

\section{MATERIALS AND METHODS}

\section{Animals}

A total of 50 female Wistar rats (Laboratory Animal Center of Shandong University, China) with an initial weight of 192-204g were used in our study. The rats were housed individually on a $12 \mathrm{~h}$ light $/ 12 \mathrm{~h}$ dark cycle at $22-24^{\circ} \mathrm{C}$ in mesh-bottom cages with free access to food and water ad libitum. The rats were acclimatized to the facilities for 7 days, and all experimental protocols were approved by the Animal Research Ethics Committee of Shandong University.

Before the study, initial cystometric investigation of the 50 rats was performed according to the method of section 2.4-cystometric investigation below, and no D0 rat was found (Figure-1a). Then, forty rats were used in an obstruction group (underwent obstruction surgical procedures) and 10 rats were used in a control group (without obstruction surgical procedures), randomly.

\section{Surgical procedures}

In the study, the 40 rats of the obstruction group were anaesthetized with chloral hydrate (350mg/kg i.p.). The urethra was exposed and the urinary bladder was catheterized with a human epidural catheter (F3, 1.0mm outer diameter) via the urethral orifice (Figures-2a and b). Care was taken to identify the urethra and vagina, and a needle with 4/0 silk suture was pierced around the perineal urethra (Figure-2c). A constant degree of partial obstruction was created by tying the silk ligature loosely around the urethra in the presence of the intraluminal indwelling catheter (Figure$-2 \mathrm{~d}$ ), and then the epidural catheter was removed (Figure-2e). The rats were observed overnight in the recovery room, and then returned to normal conditions with food and water ad libitum. Six weeks later, the ligature around urethra was removed in the obstruction rats.

All of the obstruction rats were performed by one investigator. No prophylactic antibiotic was used after the surgical procedures. Moreover, in our preliminary experiment, we dissected an obstruction rat after surgical procedures for the demonstration to identify the location of ligature (Figure-2a,b and c).

\section{Ligature removal}

Two rats died within one week after the surgical procedures in the obstruction group, and acute retention was probably the cause of death. Moreover, the rats tried to remove the ligature, and the ligature ablated in 4 rats, which were excluded from the study. Therefore, the ligature was removed in only 34 rats after 6 weeks of obstruction.

The rats were anesthetized with chloral hydrate $(350 \mathrm{mg} / \mathrm{kg}$ i.p.). The ligature around urethra (Figure-3a) was cut and carefully removed to make the stenosis disappeared and enable the transurethral cystometric investigation (Figure-2b). After the ligature around urethra was removed, the rats were allowed to recover for two days. Then, cystometric investigation was performed in the 34 obstruction rats and the 10 control rats, and the probability of DO was analyzed.

\section{Cystometric investigation}

In the cystometric investigation, the conscious 44 rats were held under partial restraint in a restraining device we designed (Figure-4) which enabled the measurement of cystometry. The bladder was catheterized through urethra by a 
Figure 1 - a) the initial cystometric investigation of the $\mathbf{5 0}$ rats before our study (two voiding contractions were observed and the number indicated peak voiding pressure). b) the cystometric investigation of the $\mathbf{1 0}$ control rats $\mathbf{6}$ weeks later (one voiding contraction was observed and the number indicated peak voiding pressure). c) the cystometric investigation of the 6 detrusor overactivity rats 6 weeks later (one voiding contraction was observed and the number indicated peak voiding pressure, the low peak voiding pressure group). d) the cystometric investigation of the 5 detrusor overactivity rats $\mathbf{6}$ weeks later (one voiding contraction was observed and the number indicated peak voiding pressure, the high peak voiding pressure group).
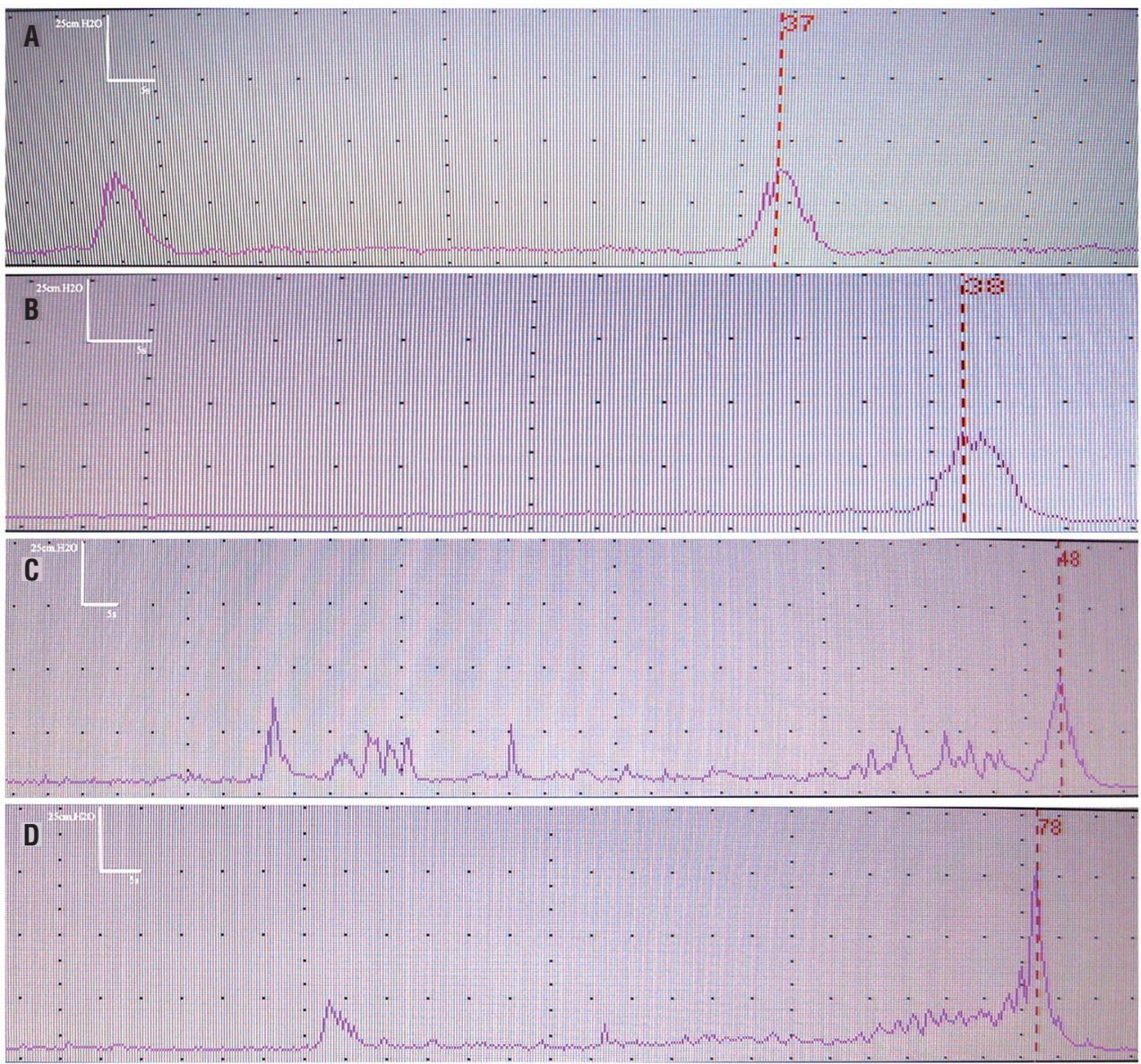

human epidural catheter (F2, 0.7mm outer diameter, $0.4 \mathrm{~mm}$ internal diameter), which was connected via a T-tube to urodynamic testing machine (Laborie medical technologies, Corp) and infusion pump (LION WZ-50C6 microinfusion pump, Zhejiang University, China).

The rat was placed supine and the urethral orifice could be observed clearly. Data were collected and analyzed after the animals were seen to be resting quietly in the restraining device (Figure-4). The cystometric investigation was performed by infusing warm saline $\left(37-38^{\circ} \mathrm{C}\right)$ at a rate of $9 \mathrm{~mL} / \mathrm{h}$, and the infusion was stopped when voiding contraction was observed according to the voiding pressure and the leakage of urine around the urethral orifice. Bladder emptying was ascertained by opening the catheter and gently pressing the lower abdomen after the cystometry. At least three voiding cycles per animal were recorded to insure reproducibility of the bladder responses. Bladder function was evaluated using the following criteria: the presence, frequency and amplitude of non-voiding contractions, bladder capacity (volume of infused saline at micturition) and peak voiding pressure (peak bladder pressure during micturition). 
Figure 2 - a, b, c, d and e: the surgical procedures for obstruction rats. A, B and C: the dissection of an obstruction rat to identify the location of ligature.
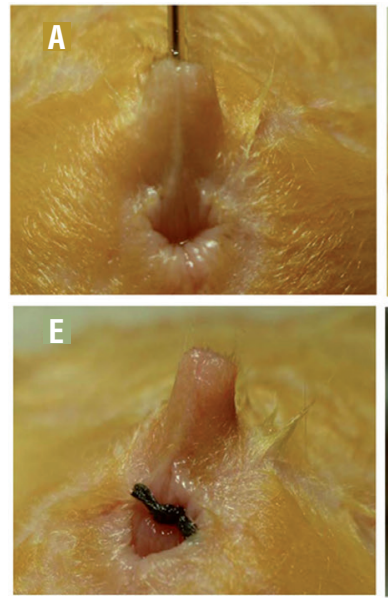
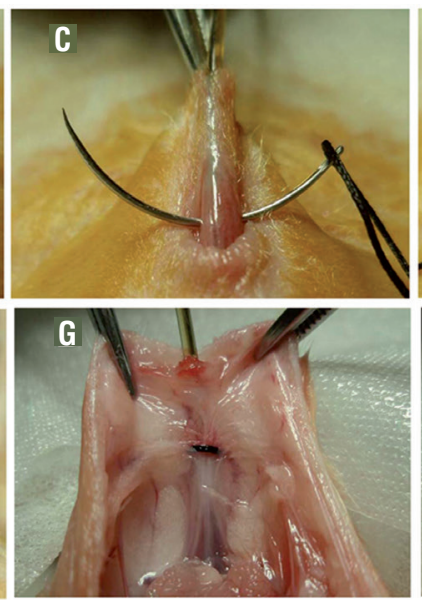

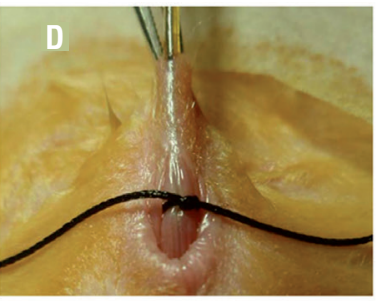

H
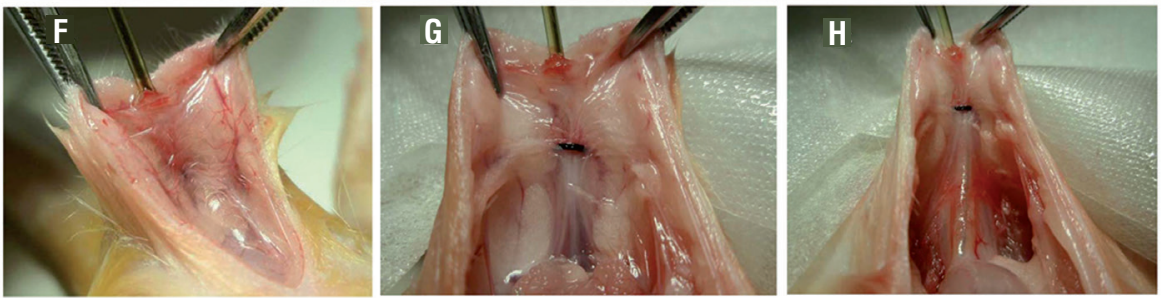

Figure 3 - a) the ligature of obstruction rats 6 weeks later. b) the intra-urethral cannula introduction was performed after the ligature removal.
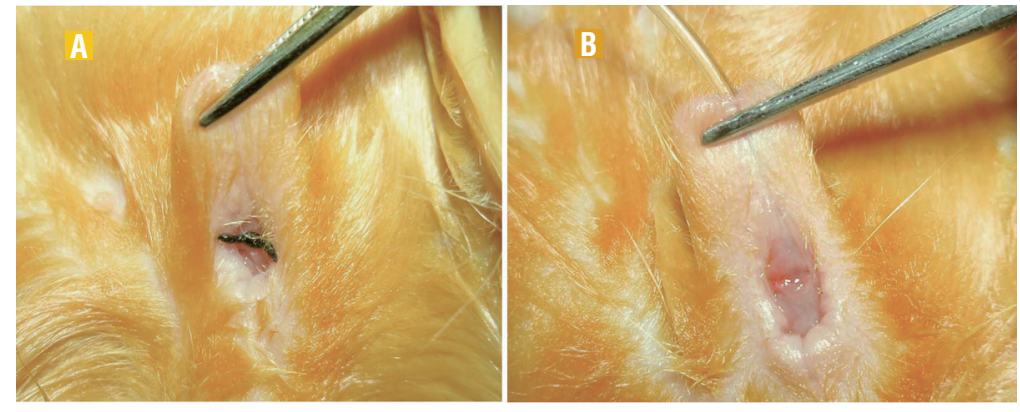

Figure 4 - The restraining device we designed which enabled cystometric investigation.

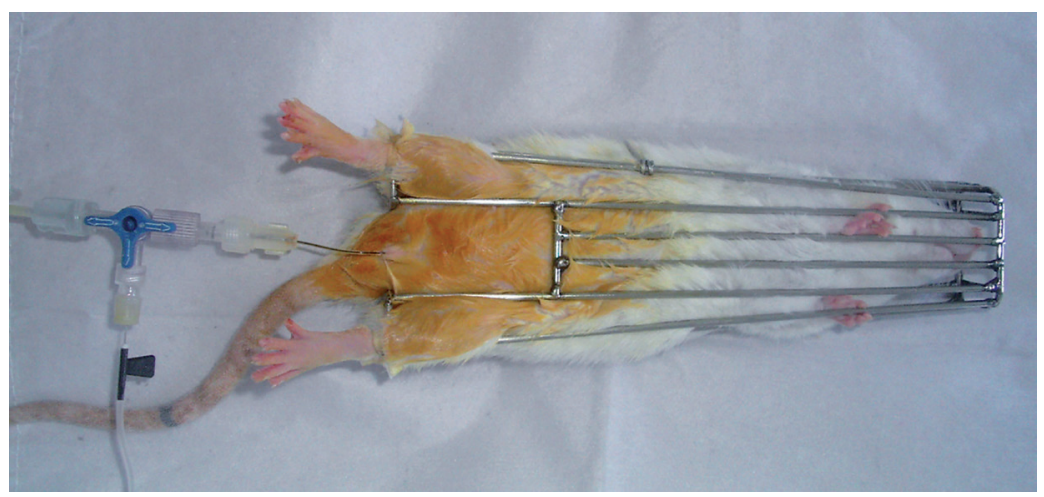


During the filling phase, some of the obstruction rats had obvious non-voiding contractions before the onset of voiding contraction and thus were defined as having DO and classified as the DO group (8-10).

\section{Data analysis}

The Statistical Package for Social Sciences was used to handle the database. To determine the relationship between a categorical variable with two levels and normally or non-normally distributed quantitative variables, Student's t-test or Mann-Whitney U tests were applied. The two-sided $\mathrm{P}<0.05$ was considered to indicate statistically significant differences.

\section{RESULTS}

Cystometric investigation was performed in the 34 obstruction rats and 10 control rats. DO ratio of the obstruction rats was 32.4\% (11 out of 34) (Figure-1c and d), and the control rats had no DO (0 out of 10) (Figure-1b). Compared to the control rats, the bladder capacity and peak voiding pressure increased significantly in the DO rats.

The bladder capacity increased from $0.273 \pm$ $0.036 \mathrm{~mL}$ in the control rats to $0.89 \pm 0.19 \mathrm{~mL}$ in the
D0 rats $(\mathrm{P}<0.001$; Table-1). The peak voiding pressure increased from $45.9 \pm 4.1 \mathrm{~cm} \cdot \mathrm{H}_{2} \mathrm{O}$ in the control rats to $63.5 \pm 17.4 \mathrm{~cm} . \mathrm{H}_{2} \mathrm{O}$ in the $\mathrm{DO}$ rats $(\mathrm{P}=0.007$; Table-1). For the obstruction group, compared to the non-D0 rats, the DO rats had higher bladder capacity $(0.89 \pm 0.19 \mathrm{~mL}$ versus $0.43 \pm 0.09 \mathrm{~mL}, \mathrm{P}<0.001$; Table-1) and higher peak voiding pressure $(63.5 \pm$ $17.4 \mathrm{~cm} . \mathrm{H}_{2} \mathrm{O}$ versus $44.8 \pm 6.2 \mathrm{~cm} . \mathrm{H}_{2} \mathrm{O}, \mathrm{P}=0.005$; Table-1). Moreover, the D0 rats were classified into the low peak voiding pressure group (Figure-1c) and the high peak voiding pressure group (Figure-1d) (49.2 $\pm 4.2 \mathrm{~cm} . \mathrm{H}_{2} \mathrm{O}$ in group 1 versus $80.8 \pm 7.1 \mathrm{~cm} \cdot \mathrm{H}_{2} \mathrm{O}$ in group 2, $\mathrm{P}<0.001$; Table-1).

Furthermore, we studied the body mass and bladder weight in the 10 control and 34 obstruction rats 6 weeks later. The bladder weight was the highest in the D0 rats, with significant differences between the D0 rats and the control rats and non-D0 rats $(\mathrm{P}=0.003, \mathrm{P}=0.028$; Table- 2 ). We harvested bladder of the control rats and the DO rats for subsequent histological assessment. Histologically, compared to the control rats, the DO rats presented an increased detrusor muscle cell mass due to hypertrophy (Figures-5 a and b). On the other hand, compared to the control rats, the DO rats detrusor muscle cell gap widened with abundant collagen fiber (Figure-6 $\mathrm{a}$ and $b$ ).

Table 1 - Comparison of cystometric investigation in the control and obstruction rats (Mean \pm SD).

\begin{tabular}{|c|c|c|c|c|c|}
\hline Groups & $n(\%)$ & $\mathrm{BC}(\mathrm{mL})$ & $P$ & $\mathrm{VP}\left(\mathrm{cm} \cdot \mathrm{H}_{2} \mathrm{O}\right)$ & $P$ \\
\hline ICl & 50 & $0.18 \pm 0.04$ & & $38.8 \pm 3.2$ & \\
\hline $\mathrm{Cl}$ & 44 & & & & \\
\hline Control & 10 & $0.273 \pm 0.036$ & $<0.001^{a}$ & $45.9 \pm 4.1$ & $0.007^{\mathrm{a}}$ \\
\hline Obstruction & 34 & & $<0.001^{b}$ & & $0.005^{\mathrm{b}}$ \\
\hline Non-D0 & $23(67.6)$ & $0.43 \pm 0.09$ & & $44.8 \pm 6.2$ & \\
\hline DO & $11(32.4)$ & $0.89 \pm 0.19$ & $0.716^{c}$ & $63.5 \pm 17.4$ & $<0.001^{c}$ \\
\hline group 1 & $6(54.5)$ & $0.91 \pm 0.21$ & & $49.2 \pm 4.2$ & \\
\hline group 2 & $5(45.5)$ & $0.87 \pm 0.19$ & & $80.8 \pm 7.1$ & \\
\hline
\end{tabular}

a: DO versus control; b: DO versus Non-D0; c: group 1 versus group 2

$\mathbf{B C}=$ Bladder capacity; $\mathbf{V P}=$ Peak voiding pressure; $\mathbf{I C I}=$ Initial cystometric investigation of the 50 female Wistar rats before the study; $\mathbf{C l}=$ Cystometric investigation 6 weeks later; $\mathbf{D O}$ = Detrusor overactivity 
Table 2 - Comparison of body mass and bladder weight in the control and obstruction rats 6 weeks later (Mean \pm SD).

\begin{tabular}{lccccc}
\hline Groups & $\mathrm{n}(\%)$ & $\mathrm{BM}(\mathrm{g})$ & $\mathrm{P}$ & $\mathrm{BW}(\mathrm{g})$ & $\mathrm{P}$ \\
\hline Control & 10 & $311 \pm 8$ & $0.244^{\mathrm{a}}$ & $0.127 \pm 0.015$ & $0.003^{\mathrm{a}}$ \\
Obstruction & 34 & & $0.500^{\mathrm{b}}$ & & $0.028^{\mathrm{b}}$ \\
Non-D0 & $23(67.6)$ & $307 \pm 13$ & & $0.159 \pm 0.041$ & \\
D0 & $11(32.4)$ & $302 \pm 24$ & $0.572^{\mathrm{c}}$ & $0.226 \pm 0.085$ & $0.111^{\mathrm{c}}$ \\
group 1 & $6(54.5)$ & $306 \pm 23$ & & $0.189 \pm 0.068$ & \\
group 2 & $5(45.5)$ & $297 \pm 27$ & & $0.271 \pm 0.087$ & \\
\hline
\end{tabular}

a: D0 versus control; b: DO versus Non-D0; c: group 1 versus group 2

$\mathbf{B M}=$ Body mass; $\mathbf{B W}=$ Bladder weight; $\mathbf{D} \mathbf{0}$ = Detrusor overactivity

Figure 5 - The frozen section of detrusor muscle cell (H\&E staining, x200). a) control group; b) detrusor overactivity group.

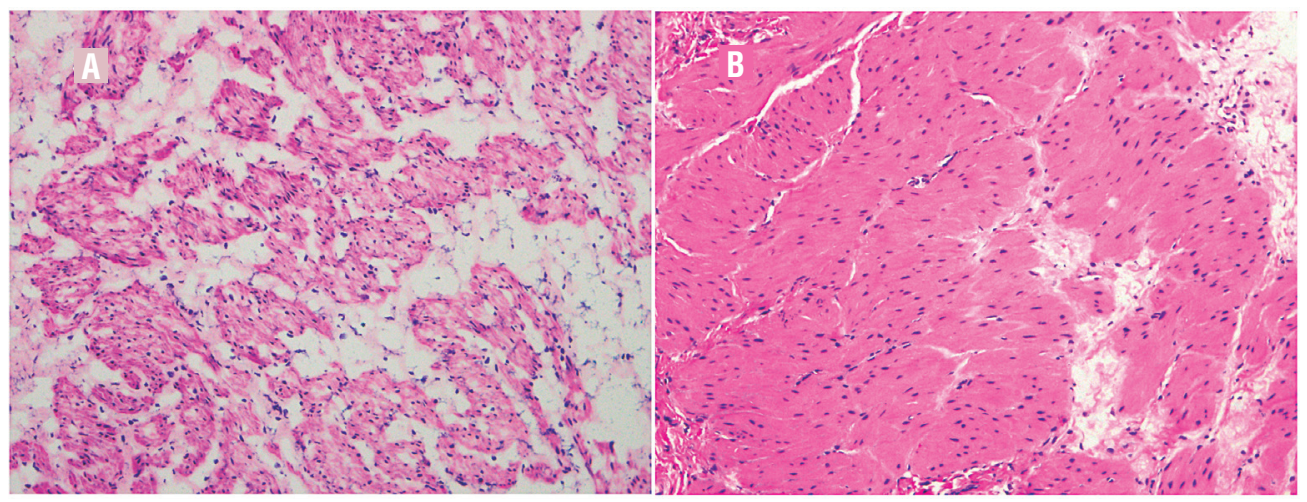

Figure 6 - The frozen section of collagen fiber (H\&E staining, x200). a) control group; b) detrusor overactivity group.
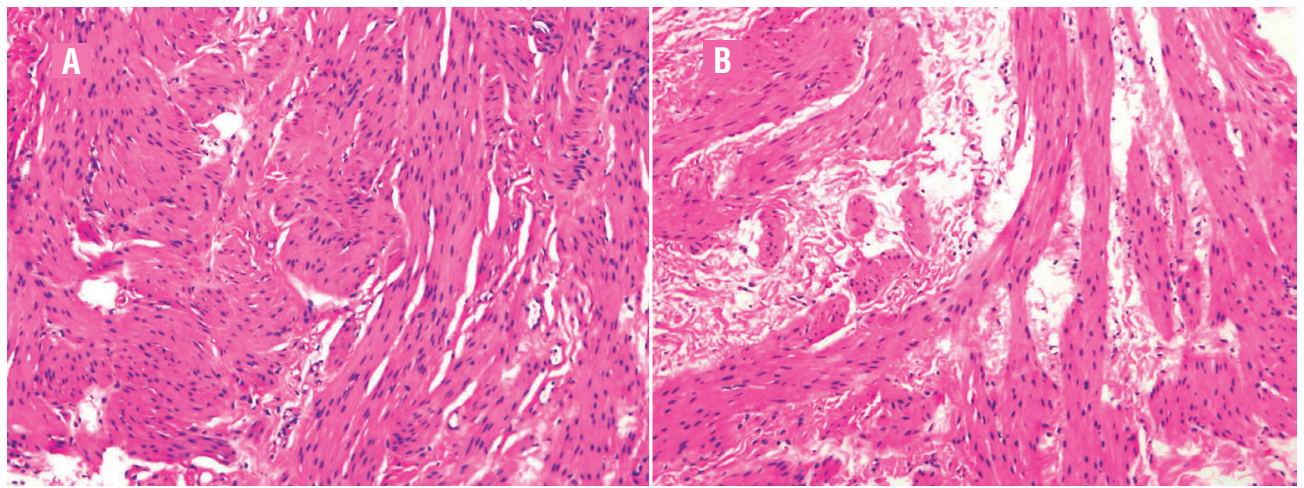


\section{DISCUSSION}

We established an improved model of DO in female Wistar rats. Our results confirmed that the obstruction-related DO rats presented altered voiding patterns. Non-voiding contractions occurring prior to voiding were observed in the DO rats. Following voiding, no involuntary detrusor contraction was recorded but the involuntary detrusor contractions developed slowly with increasing amplitude until the next voiding occurred.

In the previous study (3), four types of voiding pattern were described in the D0 rats with trans-abdominal approach, and the incidence rate was 54\% (type I, non-voiding contractions occurring before and after micturition), 26\% (type II, non-voiding contractions occurring prior to micturition), 13\% (type III, similar to that of control) and 7\% (type IV, non-voiding contractions associated with dribbling and no micturition occur), respectively. However, only one type of DO pattern was observed in our study and the bladder capacity was low. The differences between our study and the previous study may be due to the different method in establishing and evaluating DO rats.

Interestingly, the DO pattern observed in our study was consistent with one of the patterns (type II) described in the previous study (3) with approximate incidence rate (32.4\% versus $26 \%)$ and was characterized by an increased involuntary detrusor contractions during the filling phase, which was in agreement with fundings in men showing DO associated with an increase in voiding pressure or men with prostatic obstruction (11). It was conjectured that the other three types of pattern (type I, type III and type IV) may be induced by the impairment of anatomic structure in the previous study (3). Therefore, the DO model established with our method was more consistent with the natural history of obstruction-related DO.

We summarized the characteristics of the improved DO model as follows:

1. The method was simple and convenient. We used a needle with silk suture and tied perineal urethra to establish obstruction-related DO model. Moreover, the survival rate after surgical procedures was satisfactory $(38 / 40,95.0 \%)$.
2. The method did not need a low abdominal incision. The impairment of bladder and its innervation and peri-urethral blood vessel was avoided. Therefore, compared to the previous studies $(3,7)$, the influence of injury on bladder, which might be the etiology of DO, was excluded.

3. Cystometric investigation was performed without anesthesia after ligature removal in obstruction rats, and bladder was catheterized through urethra. No other operation was performed. The wound and cicatricle was reduced, and the equality and stabilization of obstruction was improved. Moreover, compared to the bladder catheter implantation $(3,7)$, cystometric investigation was performed through urethra in the improved model, which was consistent with the clinical examination for patients. In the study, although cystometric investigation was performed through urethra by catheter, no obstruction was observed during voiding.

4. The improved model had no impairment of anatomic structure and had satisfactory adaptive capacity to environment. Therefore, the model can be fed and transported easily, and bulk production is feasible.

5. It was an easily reproducible method for producing obstruction-related DO model in female Wistar rats, which could provide a continuum of tissue and urodynamic data that could be used to further study the pathophysiologic changes underlying DO. Moreover, the improved model was potentially suitable for further evaluation of mechanisms involved in the development of DO and the responses to pharmacological treatment.

However, our study had several weaknesses. Although the improved DO model characterized by an increased contractile activity in detrusor muscle during the filling phase appeared to have common features with human, the incidence rate 
of DO shown in the study was lower than the previous study (3). Moreover, the movement of rats interfered with cystometric investigation when they showed the sign of discomfort or distress, though the interference was not frequent.

In conclusion, we studied an improved model for the establishment and evaluation of DO in female Wistar rats. Ligating perineal urethra and filling cystometry with intra-urethral cannula approach is a simple and easily reproducible method to establish and evaluate the model of DO rats, which can provide a continuum of tissue and urodynamic data that can accurately reproduce the urodynamic changes observed in patients with overactive bladder and may be used to study the pathophysiologic changes underlying DO.

\section{ACKNOWLEDGMENTS}

Our study was supported by the National Natural Science Foundation of China (Grant number: 30872565). Our study was supported by the Autonomic Innovative Foundation of Shandong University (Grant number: 2012TS146).

\section{CONFLICT OF INTEREST}

None declared.

\section{REFERENCES}

1. Abrams P, Cardozo L, Fall M, Griffiths D, Rosier P, Ulmsten $U$, et al.: The standardisation of terminology of lower urinary tract function: report from the Standardisation Sub-committee of the International Continence Society. Neurourol Urodyn. 2002; 21: 167-78.
2. Haylen BT, de Ridder D, Freeman RM, Swift SE, Berghmans $B$, Lee J, et al.: An International Urogynecological Association (IUGA)/International Continence Society (ICS) joint report on the terminology for female pelvic floor dysfunction. Neurourol Urodyn. 2010; 29: 4-20.

3. Lluel P, Duquenne C, Martin D: Experimental bladder instability following bladder outlet obstruction in the female rat. J Urol. 1998; 160: 2253-7.

4. Brading AF: A myogenic basis for the overactive bladder. Urology. 1997; 50: 57-67; discussion 68-73.

5. Oh MM, Choi H, Park MG, Kang SH, Cheon J, Bae JH, et al.: Is there a correlation between the presence of idiopathic detrusor overactivity and the degree of bladder outlet obstruction? Urology. 2011; 77: 167-70.

6. Oelke M, Baard J, Wijkstra H, de la Rosette JJ, Jonas U, Höfner $\mathrm{K}$ : Age and bladder outlet obstruction are independently associated with detrusor overactivity in patients with benign prostatic hyperplasia. Eur Urol. 2008; 54: 419-26.

7. Malmgren A, Sjögren C, Uvelius B, Mattiasson A, Andersson $\mathrm{KE}$, Andersson PO: Cystometrical evaluation of bladder instability in rats with infravesical outflow obstruction. J Urol. 1987; 137: 1291-4.

8. Elzayat E, Khaled S, Kashiwabara T, Elhilali M, Corcos J: Effect of the potassium channel opener WAY-133537 on the overactive bladder of spinalized rats. Neurourol Urodyn. 2006; 25: 808-14.

9. Abrams P: Describing bladder storage function: overactive bladder syndrome and detrusor overactivity. Urology. 2003; 62: 28-37; discussion 40-2.

10. Dion SB, Zvara P, Tu LM, Richer M, Corcos J: Evaluation of the role of neurolinins and urecholine hypersensitivity in an animal model of infravesical outflow obstruction. Urology. 1998; 52: 909-14.

11. Cucchi A, Achilli MP, Ravasi S, Arrigoni N: Detrusor instability as an energy-saving device in prostatic obstruction. J Urol. 1997; 157: 866-70.

Correspondence address: Jun Chen, MD Department of Urology, Qilu Hospital,Shandong University 107\#, Wenhua Xi Road, Jinan, 250012, P.R. China Telephone: + 860531 8216-6711 E-mail:znz95znz@mail.sdu.edu.cn 
I am particularly happy to see a manuscript that attempts to create an experimental model for the study of overactive bladder. We know that this disease still lacks adequate animal models and previously published models have potential risk of injury to the bladder nerves of experimental animals adopting a transabdominal approach.

As the authors mentioned, the movement of rats interfered with cystometric investigation when they showed the sign of discomfort or dis- tress, and that can hence loss results reliability. Another fact of concer is the low incidence rate of D0 shown in the study.

I believe, however, that even seeming a simple model, only time will tell if this method is easily reproducible in other research centers.

João Antonio Pereira Correia, MD Chief of Urogynecology Department Brazilian Society of Urology, Rio de Janeiro section E-mail: joaoapc@ig.com.br 